\title{
FORUM
}

\section{Transformation, Konfliktbewältigung und Integration - die europäische Perspektive der Republik Moldau zwischen Georgienkrise und Parlamentswahlen}

\author{
Manfred Grund und Hans Martin Sieg*
}

Die Republik Moldau hat mit größeren Belastungen zu kämpfen als jedes andere europäische Land westlich von Russland. Dazu zählen die wirtschaftlichen Strukturschwächen des ärmsten Landes in Europa ebenso wie der nach wie vor ungelöste, frozen conflict ' um das abtrünnige Regime in Transnistrien. Umso größer ist die Bedeutung einer europäischen Perspektive sowohl für den wirtschaftlichen Aufbau in der Republik Moldau, ihre politische Transformation sowie eine künftige Wiedererlangung der staatlichen Einheit. Der deutsche Bundestag hat die europäische Integration der Republik Moldau deshalb mit einer Resolution ausdrücklich unterstützt. ${ }^{1}$

Im Augenblick sind es vor allem zwei Anlässe, die die Aufmerksamkeit auf die Republik Moldau lenken. Dazu zählen erstens die für das kommende Frühjahr anstehenden Parlamentswahlen, die zu einer entscheidenden Wegmarke für die innere Entwicklung des Landes und seine europäischen Ambitionen werden können. Zweitens wirft die Krise in Georgien die Frage nach möglichen Rückwirkungen auf den Transnistrienkonflikt auf. Könnte eine entschiedenere Strategie Moskaus auch dort eine Verhärtung der Fronten zur Folge haben, den Unabhängigkeitsbestrebungen in Tiraspol Auftrieb verleihen oder sogar zu einer russischen Anerkennung führen, wie im Falle Abchasiens und Südossetiens? Oder handelt es sich doch um unterschiedlich gelagerte Konfliktkonstellationen, die im Falle Moldaus bessere Chancen für eine friedliche Konfliktbewältigung eröffnen? Im Folgenden wird erörtert, welche Bedeutung der europäischen Integration für beide Aspekte, den Transformationsprozess in Moldau und den Transnistrienkonflikt, zukommt.

\section{Die Rolle der Europäischen Union}

Es war in erster Linie der ungelöste Transnistrienkonflikt, der verhinderte, dass Moldau eine Beitrittsperspektive erhalten konnte. Insofern ist der bisherige Ausschluss Moldaus aus dem Erweiterungsprozess letztlich nur historischen und politischen Zufällen geschuldet. Die baltischen Länder, die ebenso wie Moldau mit dem Hitler-Stalin-Pakt an die Sowjetunion fielen, sind bereits seit Jahren Mitglieder der Europäischen Union. Im Vergleich zum Westlichen Balkan bewirkte im Falle Transnistriens gerade das vergleichsweise frühe Ende des bewaffneten Konfliktaustrages, dass Moldau noch nachträglich in den Stabilitätspakt für Südosteuropa aufgenommen wurde, aber keine Beitrittsperspektive mehr erhielt.

\footnotetext{
* Manfred Grund, MdB, Parlamentarischer Geschäftsführer der CDU/CSU-Fraktion, Mitglied des Auswärtigen Ausschusses und Länderbeauftragter des Bundestages für die Republik Moldau.

Dr. Dr. Hans Martin Sieg, außen- und sicherheitspolitischer Fachreferent bei MdB Grund und Lehrbeauftragter der Freien Universität Berlin sowie der Universität Passau.

1 Antrag der Fraktionen CDU/CSU, SPD, FDP und BÜNDNIS 90/DIE GRÜNEN. Die europäische Integration der Republik Moldau unterstützen, Bundestagsdrucksache 16/9755, angenommen am 26.06.2008.
} 
Doch während der Transnistrienkonflikt einer Beitrittsperspektive Moldaus im Wege stand, hat er inzwischen zu einem verstärkten Engagement der Europäischen Union geführt. Zwar wurde mit Moldau, wie mit den meisten anderen ehemaligen Sowjetrepubliken, bereits Ende der Neunzigerjahre ein Partnerschafts- und Kooperationsabkommen (PKA) geschlossen. Auch war Moldau ein primärer Adressat der Nachbarschaftspolitik, mit einem ersten Aktionsplan von Anfang 2005 und mit einer dreijährigen anstatt der sonst meist üblichen fünfjährigen Geltungsdauer. Vor allem aber intensivierte die Europäische Union ihr Engagement, nachdem es Russland Ende 2003 beinahe gelungen war, mit dem sogenannten KozakMemorandum eine Regelung in seinem Sinne durchzusetzen.

Im März 2005 ernannte die Europäische Union einen eigenen Sonderbeauftragten für die Republik Moldau und insbesondere für den Transnistrienkonflikt. Im September desselben Jahres schaltete sich die Union zusammen mit den USA als Beobachter in den Verhandlungsrahmen der OSZE zu Transnistrien im seitherigen 5+2 Format ein (Moldau, Transnistrien, Russland, Ukraine, OSZE + EU und USA). Nachdem die orange Revolution in der Ukraine die politischen Voraussetzungen dafür geschaffen hatte, entsandte die Europäische Union im November 2005 eine Mission zur Unterstützung der Kontrollen an der ukrainischmoldauischen Grenze. Sie dient nicht nur dem Ziel, Schmuggel und ähnliche Formen grenzüberschreitender Kriminalität einzudämmen. Da legale Exporte eine Registrierung des Exporteurs in Moldau zur Voraussetzung haben, soll so auch die moldauische Souveränität über Transnistrien unterstrichen werden. Derzeit stehen Verhandlungen über ein Nachfolgeabkommen für das in 2008 auslaufende PKA an, die sich vor allem aufgrund der anstehenden Parlamentswahlen verzögern.

In der Zusammenarbeit zwischen der Republik Moldau und der Europäischen Union konnten in den vergangenen Jahren stetig Fortschritte erzielt werden. Doch ist die Entwicklung der Beziehungen für beide Seiten hinter den Erwartungen zurückgeblieben. Für Moldau steht der Wunsch einer Beitrittsperspektive im Vordergrund. Doch auch wenn in den vergangenen Monaten wichtige Schritte wie der Abschluss eines Visaerleichterungs- und Rückführungsabkommen sowie die Gewährung autonomer Handelspräferenzen erreicht wurden, drängte Moldau bislang vergeblich auf den Beginn von Verhandlungen für ein Nachfolgeabkommen für das PKA.

Auch für die Europäische Union ist die Bilanz gemischt. Der insgesamt positiven Würdigung der Fortschritte im jüngsten Kommissionsbericht zur Implementierung der Nachbarschaftspolitik stehen nicht nur eine Reihe fortbestehender Defizite gegenüber. ${ }^{2}$ Vor allem haben sich die Hoffnungen auf eine Lösung des Transnistrienkonfliktes nicht erfüllt. Die Ursache hierfür lag nicht in mangelnder Konzessionsbereitschaft Moldaus, sondern in der fehlenden Bewegungsbereitschaft in Tiraspol und vor allem in Moskau. Daran zeigte sich aber auch, dass das Gewicht der Europäischen Union allein eine Konfliktlösung nicht herbeizuführen vermag. Obgleich die Europäische Union ihre Handlungsmöglichkeiten keineswegs ausgeschöpft hat, dürfte die Tragweite ihres politischen Engagements letztlich begrenzt bleiben - auch da der Gemeinsamen Außen- und Sicherheitspolitik (GASP) die Handlungsfähigkeit eines einheitlichen Akteurs nach wie vor fehlt. Umso größere Bedeutung erlangt damit aber die Integrationsperspektive, die sie anbieten kann; denn nur sie eröffnet Moldau langfristig eine verlässliche Alternative zu einem fragwürdigen Arrangement mit Moskau und Transnistrien und kann zugleich den Fortschritt des Transformationsprozesses sicherstellen.

2 Europäische Kommission: Commission staff working document. Accompanying the Communication from the Commission to the Council and the European Parliament. Implementation of the European neighbourhood policy in 2007. Progress Report Moldova, SEC(2008) 399. 
Allerdings bleibt zwischen Moldau und der Europäischen Union eine Asymmetrie der Interessenlagen zu konstatieren. Moldau verbindet mit der europäischen Integration vitale Interessen: die Überwindung von Trennlinien zum übrigen Europa, wirtschaftliche Wachstumsperspektiven, neue Absatzmärkte für den durch russische Importverbote schwer geschädigten Export, außenpolitische Anlehnung und Unterstützung im Blick auf den Transnistrienkonflikt. Das Interesse der Europäischen Union an Moldau wird hingegen stärker durch abstrakte oder normative Erwägungen konstituiert. Im Kern liegt es im Leitbild der europäischen Integration selbst begründet; denn schon angesichts der bestehenden Mitgliedschaften und Beitrittsperspektiven wird sich prinzipiell nicht dauerhaft rechtfertigen lassen, Moldau von den Vorteilen des Einigungsprozesses auszuschließen.

Aber auch sicherheitspolitisch kann die Europäische Union kein Interesse daran haben, dass Moldau, das neben vielen anderen Problemen mit einer massiven Abwanderung an potenziellen Leistungsträgern zu kämpfen hat, auf Dauer zum Armen- und Altenheim in Europa wird; denn damit gingen zumindest potenzielle Risiken für die demokratische Entwicklung und die gesellschaftliche und regionale Stabilität einher. Vor allem aber verbinden sich konkrete Risiken mit Transnistrien. Als Ausgangsort eines regionalen Konfliktes, als möglicher Ausgangspunkt für organisierte Kriminalität und Waffenschmuggel ${ }^{3}$ oder sogar als potenzieller Rückzugsort für Terroristen bildet Transnistrien und letztlich die Natur des dortigen Regimes die in der europäischen Sicherheitsstrategie von 2003 identifizierten „Hauptbedrohungen" gleich in mehrfacher Hinsicht ab. ${ }^{4}$

Das Interesse der Europäischen Union an Moldau folgt daher zwei Motiven, die sich gegenseitig bedingen, aber nicht gegeneinander aufrechnen lassen: Einer Lösung des Transnistrienkonfliktes und einer Fortsetzung des Transformationsprozesses, der damit auch Transnistrien erfassen muss. Die Tragfähigkeit einer Strategie, die beide Ziele miteinander verbindet, wird entscheidend von der europäischen Perspektive für Moldau abhängen.

\section{Innenpolitische Aspekte}

Der jüngste Fortschrittsbericht der Kommission dokumentiert mit seinem insgesamt positiven Zwischenfazit den Willen zur europäischen Integration, der sich parteiübergreifend in allen größeren politischen Kräften in Moldau feststellen lässt. ${ }^{5}$ Defizite liegen weit weniger in der Gesetzgebung als vielmehr in deren Implementierung. In dieser Hinsicht vollzieht sich der Transformationsprozess in der Republik Moldau unter ähnlichen Schwierigkeiten wie in mancher anderen ehemaligen Sowjetrepublik. Er erschöpft sich nicht in politischen, administrativen und wirtschaftlichen Reformen allein. Er bedarf ebenso eines Wandels von Gewohnheiten und Mentalitäten sowie einer Entflechtung wirtschaftlicher, administrativer und politischer Strukturen, die sich oft informell fortsetzen.

So ist Korruption nach den einschlägigen Indikatoren von Weltbank und Transparency International ein ebenso weitverbreitetes wie tief greifendes Problem in Moldau. ${ }^{6}$ Zwar wurden die rechtlichen Grundlagen weitgehend internationalen Standards angepasst. ${ }^{7}$ Doch ob-

3 Verdachtsmomente, nach denen Transnistrien eine Drehscheibe für den Waffenschmuggel geworden ist, konnten bislang zwar nicht in größerem Umfang bestätigt werden. Dabei sollte jedoch die Effektivität der Grenzkontrollen nicht überschätzt werden.

4 Europäischer Rat: Ein sicheres Europa in einer besseren Welt. Europäische Sicherheitsstrategie, Brüssel 2003, S. 3-4.

5 Europäische Kommission: Progress Report Moldova, 2008.

6 Transparency International: Report on the Transparency International Global Corruption Barometer 2007, Berlin 2007, S. 21-24; World Bank: The Worldwide Governance Indicators, Country Data Report for MOLDOVA, 1996-2007, S. 7.

7 So wurden 2007 sowohl die UN-Konvention gegen Korruption als auch das Zusatzprotokoll zur Strafrechtskonvention des Europarates gegen Korruption ratifiziert. 
wohl Moldau eine eigene Strafverfolgungsbehörde zur Bekämpfung der Korruption eingerichtet hat, besteht auch hier ein Problem in der konsequenten Durchsetzung der Rechtsnormen. Eine objektive Einschätzung dieser Problematik wird zudem durch den Umstand erschwert, dass Anklagen wegen Korruption in verschiedenen Fällen den Verdacht politischer Instrumentalisierung begründen konnten, ${ }^{8}$ wie beispielsweise im Falle von Serafim Urechean, des früheren Chisinauer Bürgermeisters und jetzigen Vorsitzenden der Partei „Unsere Moldau“.

Zugleich leidet das Justizsystem unter einer mangelnden materiellen und personellen Ausstattung sowie zuweilen fehlender Professionalität. ${ }^{9}$ Hinzu kommt, dass die Unabhängigkeit der Justizbehörden in der Verfassungswirklichkeit begrenzt geblieben ist, die Befugnisse der Strafverfolgungsbehörden, vor allem der Generalstaatsanwaltschaft, aber weder klar umrissenen Beschränkungen noch hinreichenden Kontrollmechanismen unterliegen. Der damit verbundene Mangel an rechtsstaatlichen Garantien beeinträchtigt beispielsweise die Investitionssicherheit, in Bezug auf die Zustände in Gefängnissen und im Polizeigewahrsam aber auch grundlegendere europäische Menschenrechtsnormen. ${ }^{10}$ Nicht zuletzt ist damit auch ein unzureichender Schutz vor politischen Eingriffen in die Justiz verbunden.

Die legislativen und administrativen Reformen, die in den letzten Jahren und Monaten zur Stärkung der Rechtsstaatlichkeit ergriffen wurden, müssen ihre praktische Wirksamkeit erst noch beweisen. In der Realität lässt insbesondere der Einfluss des Präsidenten und seines Apparates die Grenzen zwischen den Gewalten verschwimmen. Der Verdacht der politischen Instrumentalisierung von Strafverfahren ist jedenfalls in bestimmten Fällen nicht gänzlich von der Hand zu weisen. Oppositionspolitiker und NGOs beklagen immer wieder Einschüchterungsversuche durch Justiz und Sicherheitsorgane gegenüber Oppositionellen und Medienvertretern.

Auch für die Medien bleibt eine mangelnde Unabhängigkeit von politischen Einflussnahmen festzustellen. In dieser Hinsicht konstatiert der Fortschrittsbericht der Kommission zwar ebenfalls, dass die erforderlichen Rechtsgrundlagen weitgehend geschaffen worden sind, bemängelt aber deutlich deren Umsetzung. ${ }^{11}$ So vermochte die Regierung etwa die Sendeanstalten bislang durch Lizenzvergaben zu beeinflussen. Freedom House stufte Moldau auch deshalb als nur ,,bedingt frei“ ein. ${ }^{12}$

Diese hinsichtlich der demokratischen Transformation verbliebenen Defizite stellen jedoch weniger einen Grund gegen, sondern vielmehr für eine möglichst weitgehende Integrationsperspektive dar. Denn die mit EU-Standards dann auch verbundene Konditionierung bildet ein entsprechendes Gegengewicht zu den Vorteilen, die aus den Möglichkeiten zum Missbrauch politischer Macht erwachsen könnten. Auch für die Republik Moldau bedeutet die EU-Integration einen Anreiz zur Beschleunigung des Transformationsprozesses. Weit wichtiger als der Umfang von Hilfsmaßnahmen ist dabei die sich bietende Entwicklungsperspektive. Je attraktiver und konkreter diese sich darstellt, desto eher werden sich auf Statusquo-Interessen beruhende Widerstände in Bevölkerung und Eliten überwinden lassen.

8 U.S. Department of State: Moldova. Country Report on Human Rights Practices 2007, 2008, S. 10.

9 OSCE Mission to Moldova: Analytic Report of the Trial Monitoring Programme for the Republic of Moldova: Observance of Fair Trial Standards and Corresponding Rights of Parties during Court Proceedings (April 2006 - May 2007), 2008.

10 Dies dokumentieren neben verschiedenen Urteilen des Europäischen Gerichtshofs für Menschenrechte auch Berichte von Amnesty International. Vgl. Amnesty International: Moldova. Police torture and ill-treatment: "It's just normal.", Index-Nr. EUR 59/002/2007.

11 Europäische Kommission: Progress Report Moldova, 2008, S. 5.

12 Freedom House: Freedom in the World - Moldova, 2008. 
Derartige Impulse sind aber schon deshalb dringlich, weil Zeitverluste im Transformations- und Integrationsprozess für Moldau mit hohen Kosten verbunden sein werden. Die Anziehungskraft der Europäischen Union hat Abwanderungstendenzen nach der jüngsten Erweiterungsrunde noch weiter verstärkt, vor allem in Form eines massiven Andrangs auf die rumänische Staatsbürgerschaft. Dieser dramatische „brain drain“ droht in Moldau die wirtschaftlichen Entwicklungschancen massiv zu beeinträchtigen, schon aufgrund seiner Auswirkung auf die Zusammensetzung der Wählerschaft aber auch politisch eher Beharrungskräfte zu stärken. Moldau könnte zur großen Verliererin des europäischen Einigungsprozesses werden.

\section{Die Parlamentswahl 2009}

Die Parlamentswahlen im März 2009 werden für die kommenden Jahre zum entscheidenden Prüfstein für den Demokratisierungsprozess und damit auch für die europäische Integration der Republik Moldau werden. Eine wirklich faire Wahl würde den europäischen Bestrebungen Moldaus einen nachhaltigen Schub verleihen und für die Ausgestaltung des künftigen Verhältnisses zur Europäischen Union von maßgeblicher Bedeutung sein. Sie wird gleichzeitig zu den Verhandlungen über ein Nachfolgeabkommen zum PKA stattfinden und damit über die von der Europäischen Union zu eröffnenden Integrationsangebote und -perspektiven mit entscheiden.

Dafür wird ihr Verlauf jedoch deutliche Fortschritte gegenüber den letzten Parlamentswahlen von 2005 aufweisen müssen. Damals waren die Wahlbeobachter der OSZE zwar zu dem Urteil gelangt, dass die Parlamentswahlen ,generally complied with most OSCE commitments, Council of Europe and other international standards for democratic elections". Sie hatten zugleich jedoch eine Vielzahl von Defiziten aufgezählt, insbesondere Ungleichheit bei den Wahlkampfbedingungen und dem Zugang zu den Medien. ${ }^{13}$

Neben der Chancengleichheit wird es aber auch auf eine größtmögliche Transparenz im Wahlverlauf ankommen. Denn politische Konsequenzen können sich nicht nur aus der subtilen Abwägung von Wahlkampfbeobachtern ergeben, sondern ebenso aus dem oft sehr viel plakativeren Eindruck, der die öffentliche Meinung bestimmt. In diesem Zusammenhang sei nur an die georgischen Präsidentschaftswahlen von 2008 erinnert, bei der die OSZE-Wahlbeobachter ein ähnliches Gesamturteil fällten, die Presseberichterstattung im Ausland und insbesondere in Deutschland aber primär die Kritikpunkte und Manipulationsvorwürfe der Opposition aufgriff. Tatsächlich hatte Georgien im Vergleich zu früheren Wahlen bei allen Defiziten deutliche Fortschritte erzielt, die Wähler hatten zum ersten Mal eine Alternative und auch die georgische Regierung bemühte sich nach Aussagen von Wahlbeobachtern im Vorfeld ernsthaft darum, kritisierte Vorkommnisse zu beseitigen. ${ }^{14}$ Nach dem Medienecho musste sich jedoch der Eindruck verfestigen, es habe sich um einen Rückschlag für die Demokratisierung des Landes gehandelt. Für Moldaus europäische Bestrebungen käme eine ähnliche Entwicklung einem Fiasko gleich.

Die Parlamentswahl in Moldau wird ohnehin in einem Klima tiefen Misstrauens zwischen der regierenden kommunistischen Partei und der Mehrzahl der Oppositionsparteien stattfinden. Zugleich ist das Wähler- wie auch das Parteienspektrum in Bewegung. Die kommunistische Partei muss mit der Möglichkeit rechnen, dass die Regierungsmehrheit verloren

13 OSCE, Office for Democratic Institutions and Human Rights: Republic of Moldova. Parliamentary Elections 6 March 2005. OSCE/ODIHR Election Observation Mission Final Report, Warschau 2005, S. 1ff.

14 Vgl. OSCE, Office for Democratic Institutions and Human Rights: Georgia. Extraordinary Presidential Election 5 January 2008. OSCE/ODIHR Election Observation Mission Final Report, Warschau 2008. 
geht. Die Oppositionsparteien könnten nicht zuletzt von der Unzufriedenheit vieler Wähler profitieren, ihnen fehlt jedoch teilweise der organisatorische Aufbau der Kommunisten. Zustimmung und Zusammenhalt erlangen sie weniger aufgrund ihrer programmatischen Ausrichtung. Ihre öffentliche Wahrnehmung konzentriert sich vielmehr sehr viel stärker auf das jeweilige Führungspersonal. Diese Konstellation bietet eine Vielzahl von Angriffspunkten. Vor diesem Hintergrund wird der Frage entscheidende Bedeutung zukommen, in welchem Maße die Regierungspartei ihren Einfluss auf Staatsorgane und Medien vor und während des Wahlkampfes einseitig instrumentalisiert.

Kritik mussten in diesem Zusammenhang bereits Änderungen der Wahlgesetzgebung hervorrufen, die vom moldauischen Parlament im April beschlossen wurden. Dies gilt insbesondere für Einschränkungen zur Bildung von Parteienblöcken und die Anhebung der Sperrklausel von vier auf sechs Prozent. Ein Problem besteht dabei weniger in den Regelungen an sich, die in anderen EU-Staaten wie Deutschland in ähnlicher Form bestehen als vielmehr darin, dass diese ohne Zustimmung des überwiegenden Teiles der Opposition und zugleich so kurzfristig erfolgten, dass dieser nur wenig Zeit zur Anpassung bleibt. Dies legt den Verdacht nahe, dass diese Änderungen vor allem einer Schwächung der Opposition und damit dem Machterhalt der kommunistischen Partei dienen sollen. Hinzu kommt, dass Doppelstaatsbürger von bestimmten öffentlichen Ämtern einschließlich Parlamentsmandaten ausgeschlossen werden sollen, was vorrangig in Sorgen um die nationale Identität und Loyalität begründet liegt, aber eine unverhältnismäßige Einschränkung von Bürgerrechten darstellt.

Diese Wahlrechtsänderungen wurden EU-seitig bei dem letzten Treffen des Moldau-Kooperationsrates und darüber hinaus auch von Außenkommissarin Ferrero-Waldner wiederholt deutlich kritisiert. ${ }^{15}$ Auch die Tatsache, dass bislang noch kein Mandat für die Aufnahme der Verhandlungen über ein Nachfolgeabkommen für das auslaufende PKA erzielt werden konnte, ist nicht nur auf die Absicht zurückzuführen, den Verlauf der entsprechenden Verhandlungen mit der Ukraine abzuwarten, die insofern Modellcharakter für eine vertiefte Nachbarschaftspolitik annehmen könnten. Vielmehr ist diese Verzögerung primär dem Motiv zuzuordnen, zunächst einmal die Vorbereitung der Parlamentswahl abzuwarten, somit also ein Druckmittel nicht aus der Hand zu geben.

Allerdings dürfte die Bereitschaft, auf Kritik einzugehen, in erster Linie von den in Aussicht gestellten Integrationsangeboten abhängen. Da substanzielle Verhandlungen realistischerweise kaum vor der Parlamentswahl beginnen können, würde eine vorherige Mandatserteilung der kommunistischen Partei zwar einen wichtigen Erfolg bescheren, zugleich jedoch den Anreiz für regelkonformes Verhalten im Wahlkampf erhöhen; denn je deutlicher sich Integrationsperspektiven bereits im Voraus konkretisieren, desto schwerer wiegen werden sonst im Nachhinein die negativen Folgen für das Verhältnis mit den europäischen Partnern.

Die Strategie der Europäischen Union sollte daher nicht vorrangig darauf beruhen, Konditionen für eine Mandatserteilung zu formulieren und den Verlauf der Wahlen abzuwarten, zumal angesichts der bisherigen Reformpolitik in Moldau auch kaum zu rechtfertigen ist, dass die Ausgestaltung der Beziehungen zur Europäischen Union hinter diejenigen zur Ukraine zurückfällt. Vielmehr sollte die Europäische Union bereits vor den Wahlen eine möglichst weitreichende Integrationsperspektive in Aussicht stellen und damit deutlich machen, dass Beeinträchtigungen des demokratischen Verlaufs der Wahlen zwangsläufig umso einschneidendere Konsequenzen haben. Die Europäische Union hat durch eine Intensivierung

15 Slowenische Ratspräsidentschaft der EU: Slovenian Foreign Minister Rupel heads EU-Moldova Cooperation Council, Pressemitteilung, 27.05.2008. 
ihres Engagements mit Moldau vor den Wahlen nicht viel zu verlieren. Sie sorgt damit nur dafür, dass die Regierenden in Chisinau mehr zu befürchten hätten als nur einen Wahlverlust. Als eine Vorleistung in diesem Sinne sollte auch die Resolution des Bundestages zur europäischen Integration von Moldau zu verstehen sein. ${ }^{16}$

\section{Die Georgienkrise: Präzedenzfall für Transnistrien?}

Die Georgienkrise und die Anerkennung von Abchasien und Südossetien durch Russland hat insbesondere auch in Moldau Befürchtungen hervorgerufen, Russland könne gegenüber Transnistrien auf ähnliche Weise vorgehen, den Druck auf Moldau erhöhen oder aber noch weniger Kompromissbereitschaft zeigen als bisher. Immerhin hatte Transnistrien im September 2006 mit Duldung Moskaus ein international nicht anerkanntes , Referendum ‘ für die Unabhängigkeit und einen späteren Anschluss an die Russländische Föderation organisiert. Wie in Georgien ist Russland auch in Moldau daran interessiert, eigenen Einfluss zu wahren oder wiederzuerlangen und eine West- und vor allem eine mögliche NATO-Integration des Landes von vornherein zu verhindern. Und wie in Abchasien und Südossetien dient in Transnistrien ein starker russischer beziehungsweise russifizierter Bevölkerungsanteil zur Rechtfertigung einer Schutzfunktion Moskaus. Ob Abchasien und Südossetien jedoch tatsächlich einen Präzedenzfall für das russische Vorgehen gegenüber Moldau darstellen werden, ist fraglich.

Tatsächlich unterscheidet sich der Transnistrienkonflikt von den Konflikten um Abchasien und Südossetien in mehrfacher Hinsicht. Die Gebiete westlich und östlich des Dnjepr blicken zwar auf unterschiedliche geschichtliche Traditionen zurück, aber der Konflikt folgt nur in sehr begrenztem Umfang ethnischen oder religiösen Trennlinien. Massive Vertreibungen, wie in Abchasien, hat es daher nicht gegeben, und die Rechte der russischen Minderheit sind in Moldau umfassend gewährleistet. Ausschlaggebend für die Abspaltung Tiraspols waren nicht zuletzt wirtschaftliche Interessen, insbesondere der großen Industriekombinate, wurde doch in sowjetischer Zeit ein Großteil der moldauischen Industrie in Transnistrien angesiedelt. Diese Bedingungen bieten weit günstigere Voraussetzungen für eine Konfliktbewältigung als in den ,frozen conflicts ' Georgiens und beeinträchtigen zugleich die Legitimität der russischen Position.

Hinzu kommt, dass die strategische Ausgangslage Russlands viel ungünstiger ist. Die russische Militärpräsenz in Transnistrien ist nicht entfernt mit den Fähigkeiten der 58. Armee im Kaukasus vergleichbar. Verstärkungen müssten über die Ukraine herangeführt werden, was bei Widerstand in Kiew leicht zu einer ungeplanten und unkontrollierten Ausweitung des Konfliktes führen könnte. In Georgien kann Russland darauf setzen, durch den Konflikt Vorbehalte gegen eine schnelle NATO-Integration Georgiens bestärkt zu haben. Die Abspaltung Transnistriens voranzutreiben, dürfte in Moldau wie auch in der Europäischen Union aber absehbar den gegenteiligen Effekt haben. Die größere Nähe zur Europäischen Union, nahezu unmittelbar an deren Außengrenze, würde zwangsläufig zu einer stärkeren Involvierung der Union und entsprechenden Gegenreaktionen führen, an denen Russland eigentlich kein Interesse haben kann.

Die wirtschaftliche und militärische Kräfteverteilung zwischen NATO und Europäischer Union auf der einen und Russland auf der anderen Seite ist so ungleich, dass jede aggressi-

16 Vgl. zu diesem Begründungzusammenhang die dazu zu Protokoll gegebenen Reden von Manfred Grund (CDU/CSU), Markus Meckel (SPD), Michael Link (Heilbronn) (FDP), Diether Dehm (DIE LINKE) und Rainder Steenblock (BÜNDNIS 90/DIE GRÜNEN) in Deutscher Bundestag: Plenarprotokoll 16/172 vom 26.06.2008, S. 18377-18382. 
vere Strategie Moskaus dem Westen gegenüber von ihren psychologischen Effekten abhinge, im Kern vom Ausmaß der Konfliktbereitschaft beim Gegenüber. Auch wenn Moskau solche psychologischen Vorteile auszuschlachten versucht, wiedererlangte Stärke demonstriert und größere Entschlossenheit zeigt, ist auch der Georgienkonflikt insgesamt vermutlich eher auf die lokale Dynamik im Verhältnis zwischen Moskau, Georgien und dessen abtrünnigen Regionen zurückzuführen als auf eine allgemein offensivere Strategie Moskaus. Wirtschaftlich hat Russland bei einer dauerhaften Konfrontation mit dem Westen ohnehin weit mehr zu verlieren als es mit einem Anschluss jeder dieser abtrünnigen Gebiete zu gewinnen hätte. Eine aggressivere Politik gegenüber Moldau wäre deshalb eigentlich nur für den Fall zu erwarten, dass der Westen nahezu nicht auf das russische Vorgehen in Georgien reagiert hätte oder aber eine Konfrontation sich so weit verschärft, dass das Motiv zur Zurückhaltung wegfiele. Daher ist es sogar möglich, dass Moskau künftig eine größere Bereitschaft zu einem Entgegenkommen in Transnistrien signalisiert, um die negativen Folgen seines Vorgehens in Georgien in den Beziehungen zum Westen zu kompensieren.

Wahrscheinlicher ist jedoch, dass im Transnistrienkonflikt kein baldiger Wandel eintritt. Moskaus Unterstützung für Transnistrien liegt in einer doppelten, außen- wie innenpolitischen Interessenlage begründet. Als strategisches Faustpfand gründet sein Wert in der Chance, Druck auf Moldau auszuüben. Das Smirnov-Regime ist dabei alles andere als ein idealer Verbündeter, es gibt nur keine Alternative. Hinzu kommt jedoch, dass die Unterstützung für Transnistrien innenpolitisch immer wieder mit dem Motiv begründet wurde, die russischstämmige Bevölkerung dort schützen zu müssen. Sich von diesem Argumentationsmuster zu lösen beziehungsweise Transnistrien aus Sicht vieler Russen im Stich zu lassen, würde der russischen Regierung nicht leichtfallen. Da sich Moskau durch den Rückzug Präsident Voronins von der im Kozak-Memorandum formulierten Vereinbarung vor den Kopf gestoßen fühlte und eine Lösung in seinem Sinne nicht abzusehen ist, erscheint die Situation auch aus russischer Sicht festgefahren. Sie dürfte für den Kreml zurzeit keine Priorität haben.

\section{Konfliktbewältigung und Integration}

Der EU-Integration von Moldau käme eine Schlüsselfunktion auch für eine Lösung des Transnistrienkonflikts zu; denn nachdem die unmittelbaren Verhandlungen mit Moskau jahrelang erfolglos verlaufen sind, könnte ein Strategiewechsel naheliegen, nach dem Moldau zunächst der Westintegration Priorität einräumen würde. Damit würde Moldau zwar das Ziel einer schnellen Wiedervereinigung zurückstellen, mittelfristig jedoch die Voraussetzungen dafür verbessern.

Die Wiedervereinigung gehörte zu den zentralen Wahlversprechen Präsident Voronins. Dabei setzte er vor allem auf eine unmittelbare Verständigung mit Moskau. Das war an sich auch nicht unplausibel. Denn in langjährigen Verhandlungen hatte sich gezeigt, dass das 5+2-Format der OSZE-Verhandlungen zwar vielleicht noch den legitimatorischen Rahmen einer künftigen Konfliktregelung abgeben kann, substanzielle Fortschritte aber schon aufgrund russischer Vorbehalte in ihnen nicht zu erzielen sein dürften. Die intransparente Form der bilateralen Gespräche ${ }^{17}$ und die Tatsache, dass von Seiten des Präsidenten immer wieder vermeintliche Durchbrüche angekündigt wurden, sorgte jedoch für Skepsis, mitunter auch Verwirrung bei den westlichen Partnern und stärkte letztlich die russische Verhandlungsposition. Im Ergebnis stand 2003 mit dem sogenannten Kozak-Memorandum der Vorschlag

17 Diese werden unmittelbar zwischen den Präsidialverwaltungen geführt, russischerseits unter Federführung des stellvertretenden Sekretärs des Sicherheitsrats Yuri Zubakov. 
für ein russisch-moldauisches Abkommen, dessen Zustandekommen in letzter Minute durch Interventionen von USA und Europäischer Union verhindert wurde. ${ }^{18}$

Eine Verständigung auf der Basis des Kozak-Memorandums hätte der russischen Militärpräsenz, deren Beendigung bereits 1999 auf dem OSZE-Gipfel von Moskau mit den sogenannten Istanbul Commitments zugesichert worden war, eine dauerhafte legale Basis verschafft. Die für Transnistrien vorgesehene Autonomie hätte praktisch einen Bestandsschutz für das dortige Regime bedeutet. Eine weit überproportionale Vertretung sowohl in den Legislativ- wie Exekutivorganen eines vereinigten Moldaus hätte ihm zudem eine weitgehende Vetomacht verschafft. Diese Konstellation, mit einem starken autoritären Regime in Tiraspol und einer inmitten eines Transformationsprozesses sehr viel schwächeren Regierung in Chisinau, musste - polemisch formuliert - die Frage aufwerfen, ob das Ergebnis in einem wiedervereinigten Moldau oder in einem vergrößerten Transnistrien bestanden hätte. Als Beispiel für die Grenzen einer möglichen Kompromisslinie mit Moskau hat das KozakMemorandum bislang nicht an Aktualität verloren.

Das Grundproblem einer Konfliktlösung liegt weniger in einer Verständigung zwischen Moldau und Transnistrien. Dieses Grundproblem liegt vielmehr in der Natur des von Igor Smirnov geführten Regimes in Tiraspol; denn in den Formen einer sowjetischen Tradition und von den örtlichen Medien patriarchal verbrämt, beruht dieses im Kern auf der Herrschaft eines Industriellen-Clans. Die Menschenrechtslage wurde unter anderem vom Europäischen Parlament wiederholt scharf kritisiert. ${ }^{19}$ Da die wirtschaftliche Basis und die politische Legitimität des transnistrischen Regimes begrenzt sind, hängt seine Fortexistenz maßgeblich von der Unterstützung Russlands ab.

Aus Sicht der Europäischen Union ergeben sich aus dieser Lage folgende Schlussfolgerungen: Erstens darf der Transformationsprozess in Moldau durch eine Lösung des Konfliktes nicht beeinträchtigt werden, sondern muss den demokratischen Wandel auch in Transnistrien zur Voraussetzung haben. Nach inzwischen mehr als eineinhalb Jahrzehnten politischer Indoktrination in Transnistrien würde eine Wiedervereinigung jedoch auch ein Wählerpotenzial erschließen, das der Transformation des Landes möglicherweise nicht sehr aufgeschlossen gegenübersteht. Auch deshalb dürfte nur eine Wiedervereinigung, die unter einem klaren Vorzeichen der Westintegration erfolgt, eine Gewähr für einen ungebrochenen Fortgang des Modernisierungsprozesses in Moldau bieten.

Zweitens: Da eine Konfliktlösung kaum gegen Russland durchgesetzt werden kann, kommt es darauf an, dessen Interessen beziehungsweise deren Wahrnehmung zu verändern. Das setzt voraus, dass Moldau dem Ziel der Westintegration eine eindeutige Priorität zuerkennt. Denn ohne Konzessionsbereitschaft in Chisinau schwindet für Moskau auch der strategische Wert Transnistriens. Für eine solche Politik kommt weniger eine NATO-Mitgliedschaft infrage, die in Moldau infolge jahrelang vergeblicher Verhandlungen zunehmend diskutiert wird; denn ein solcher Schritt hätte den Nachteil, russische Widerstände zu stärken, der strategische Vorteil, der sich damit erzielen ließe, wäre ohnehin und in noch höherem Maße mit einer künftigen NATO-Integration der Ukraine verbunden. Eine entscheidende Voraussetzung wäre jedoch der Integrationswille der Europäischen Union, ohne die es Moldau an der hinreichend plausiblen strategischen Alternative fehlt.

18 Vgl. Anneli Ute Gabanyi: Die Republik Moldau im Kontext der Neuen EU-Nachbarschaftspolitik, SWP-Studie S 46/2004, S. 17-21.

19 Europäisches Parlament: Menschenrechte in Moldau und insbesondere in Transnistrien. Entschließung des Europäischen Parlaments zur Lage der Menschenrechte in der Republik Moldau und insbesondere in Transnistrien, Dok. P6_TA(2006)0099 vom 16.03.2006. 
Drittens: Die Integrationsperspektiven von Moldau einschließlich eines künftigen Beitritts explizit oder implizit von einer vorherigen Lösung des Transnistrienkonfliktes abhängig zu machen, würde Moldau einseitigem Druck Moskaus und Tiraspols ausliefern. So würde der Konflikt entweder nur verlängert oder einer fragwürdigen Lösung zugeführt. Stattdessen sollte die Europäische Union mit ihrem Engagement bewusst einen Wettbewerb der Systeme fördern. Ihre Politik würde damit auf einer Doppelstrategie beruhen: Einerseits wird durch politische und wirtschaftliche Fortschritte die Attraktivität von Moldau steigen, der Abstand des transnistrischen Regimes zu den Standards der Europäischen Union aber umso deutlicher werden. Andererseits würde die russische Unterstützung für das Pariaregime in Tiraspol über kurz oder lang nur zu einer strategischen Belastung für Moskau werden.

\section{Fazit}

Eine solche Integrationsstrategie setzt Konsequenz und Geduld voraus. Letztlich ist jedoch angesichts der Interessenlage von Moldau wie der Europäischen Union keine Alternative zu erkennen. Abgesehen von allen utilitaristischen Überlegungen wird es sich aber auf Dauer auch nicht rechtfertigen lassen, dass eines der Länder, die am längsten und nachhaltigsten unter der Teilung Europas zu leiden hatten, jetzt von den Vorteilen der europäischen Einigung ausgeschlossen bleiben soll. Weder eine verstärkte regionale Zusammenarbeit auf der Grundlage der Schwarzmeersynergie, von GUAM („Organisation für Demokratie und wirtschaftliche Entwicklung“, Mitglieder: Ukraine, Georgien, Aserbaidschan und Moldau) oder einer neuen östlichen Partnerschaft im Sinne einer Osteuropaunion kann die Integrationsperspektive kompensieren, die die Europäische Union zu bieten vermag. Vor allem im Blick auf die grundlegenden Richtungsentscheidungen, die in Moldau hinsichtlich des Transnistrienkonflikts und der Parlamentswahl von 2009 anstehen, sollte die Europäische Union daher auch die Frage einer künftigen Mitgliedschaft mit einer neuen Offenheit behandeln. Selbst wenn sie derzeit nicht imstande ist, einen weiteren Beitritt in Aussicht zu stellen, wird sie es dabei auf die Dauer auch nach dem irischen ,Nein“ zum Lissabonner Vertrag nicht bewenden lassen können.

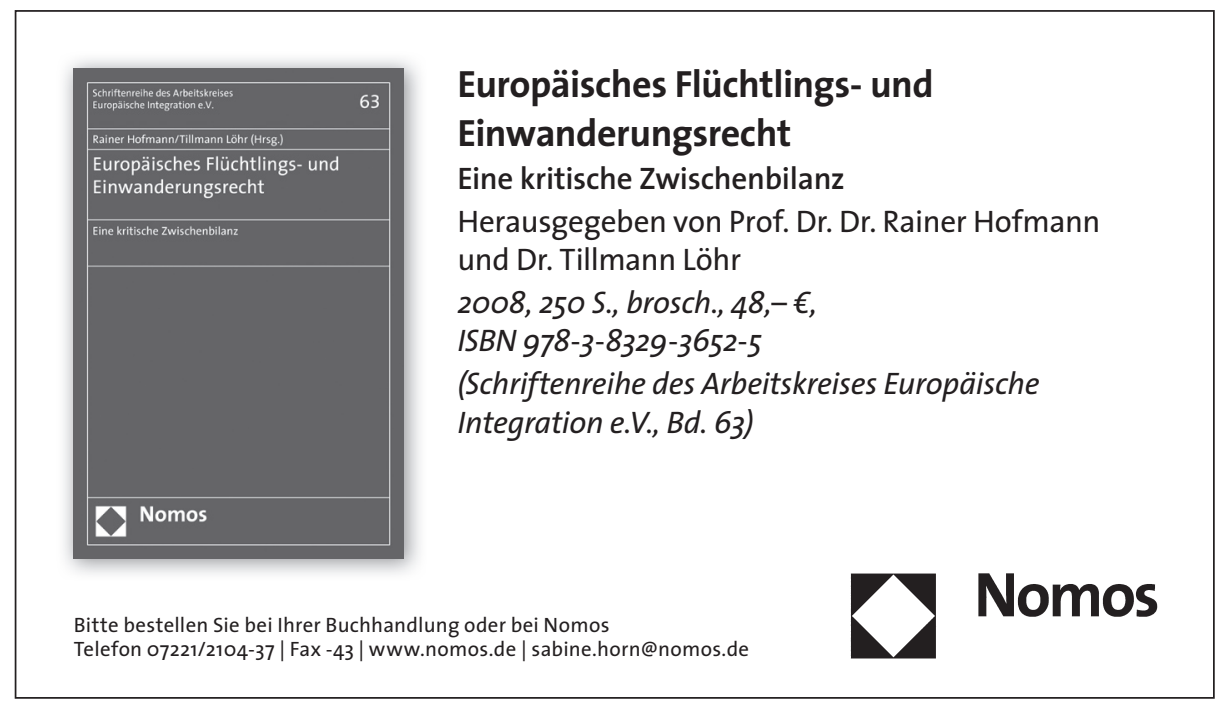

